\title{
Gradient-flipping-assisted Flux-preserving Wave Reconstruction for Improved Lowspatial- Frequency Recovery and High-contrast Imaging Using in-line Holography
}

Cigdem Ozsoy Keskinbora ( $\square$ cok@g.harvard.edu )

Max Planck Institute for Solid State Research

Wouter Van den Broek

Humboldt-Universität zu Berlin

Chris Boothroyd

Forschungszentrum Jülich

Rafal Dunin-Borkowski

Forschungszentrum Jülich

Peter van Aken

Max Planck Institute for Solid State Research

Christoph Koch

Humboldt-Universität zu Berlin

\section{Research Article}

Keywords:

Posted Date: January 27th, 2022

DOI: https://doi.org/10.21203/rs.3.rs-1267595/v1

License: (c) (i) This work is licensed under a Creative Commons Attribution 4.0 International License.

Read Full License 


\section{Abstract}

In-line holography in the transmission electron microscope is a versatile technique, which provides realspace phase information that can be used for the correction of imaging aberrations, as well as for measuring electric and magnetic fields and strain distributions. It is able to recover high-spatial-frequency contributions to the phase effectively but suffers from the weak transfer of low-spatial-frequency information, as well as from incoherent scattering. Here, we combine gradient flipping and phase prediction in an iterative flux-preserving focal series reconstruction algorithm with incoherent background subtraction that gives extensive access to the missing low spatial frequencies. A procedure for optimizing the reconstruction parameters is presented, and results from $\mathrm{MgO}$ cubes, and Fe-filled $\mathrm{C}$ nanospheres are compared with phase images obtained using off-axis holography.

\section{Introduction}

Fresnel fringes, the oscillatory features that appear at the edges of objects in images that have been recorded under (partially) coherent illumination and out-of-focus conditions, are commonly used in transmission electron microscopy (TEM) for detecting materials that consist of light elements and as a guide for focusing. They contain information about the phase of the complex exit wave that is encoded in a recorded image. Fresnel fringes are sensitive to very small relative optical path differences (phase shifts) imparted on an electron when it passes through a sample. Their analysis allows the retrieval of information about fine phase differences.

The retrieval of an electron wave function in the TEM by means of interference between a reference wave and an object wave was first proposed and demonstrated by Gabor ${ }^{1}$, who termed the technique 'holography.' Electron holography is currently applied to a wide variety of investigations ${ }^{2-9}$, which reply on the sample-imposed phase shift in an electron wave function. After the development of highly coherent electron sources by Tonomura ${ }^{10}$, holography was adapted by Leith and Upatniks ${ }^{11}$ in an off-axis configuration that helped to overcome the twin-image ${ }^{12}$ that affects the interpretation of single exposures in in-line holography. Möllenstedt ${ }^{13}$ showed that, for electrons, a charged wire can work effectively as an optical biprism, thereby allowing an off-axis reference wave to be generated in the TEM. In off-axis electron holography, the spatial resolution that can be achieved is determined in part by the interference fringe spacing ${ }^{4}$. The requirement for a high biprism voltage, microscope stability, coherence, and long acquisition times renders high spatial frequency imaging challenging for off-axis electron holography.

In-line electron holography is based on the recording of a series of images at several defocus planes, typically close to the focal plane. If the defocus is small, then interference is local, and fine Fresnel fringes carry information about high spatial frequencies in the phase. At larger defocus values, partial spatial coherence damps the contrast of Fresnel fringes because the lateral coherence length imposes an upper limit on the distance across which interference occurs. In-line electron holography is therefore efficient for recovering high spatial frequency phase information but less so for lower spatial frequencies. To a 
degree, phase changes across distances larger than the lateral coherence length can be obtained by using non-interferometric reconstruction algorithms such as the transport of intensity equation $(T I E)^{14}$. A combination of both approaches can then give access to high and low spatial frequencies in the phase ${ }^{15}$. However, in addition to amplifying low-frequency noise, the TIE is based on an elliptic partial differential equation of second order. Its solution suffers from a lack of knowledge of boundary conditions at the edges of the field of view. Iterative, nonlinear reconstruction algorithms, such as those based on initial developments by Gerchberg and Saxton ${ }^{16}$, do not suffer from the limitation of requiring well-defined boundary conditions but can require many iterations and substantial computing power to converge ${ }^{17}$.

Several iterative reconstruction algorithms have been developed based on linear and nonlinear imaging models (e.g., whether the image contrast is considered to be due to interference between an undiffracted (reference) wave and diffracted waves, or whether full interference between all partial waves transmitted by the object is taken into account). Although each algorithm has its strengths, none of them are able to recover the complete spectrum of spatial frequencies of the phase. Initializing in-line reconstruction with the phase reconstructed from an off-axis electron hologram allows the phase to be recovered at all spatial frequencies, but this approach requires a biprism to be installed in the $\mathrm{TEM}^{18}$.

Here, we introduce an in-line electron holography reconstruction algorithm that allows a wide range of spatial frequencies to be recovered by combining gradient flipping ${ }^{19,20}$ and phase prediction with incoherent background subtraction and a flux-preserving nonlinear imaging model 21,22 .

\section{li. Experimental Details}

Gradient-flipping-assisted full-resolution wave reconstruction (GF-FRWR), off-axis, and in-line electron holography experiments were carried out for two different samples. MgO cubes (Fig. 1a) and core-shell Fe-filled $\mathrm{C}$ nanospheres (Fig. 1b) were selected as test specimens. MgO cubes have previously been used in electron holography studies ${ }^{12}$. The $\mathrm{MgO}$ cubes have sharp edges, while the core-shell particles have fine features with sizes of between $\sim 0.5$ and $0.8 \mathrm{~nm}$, thereby allowing the applicability of the method to be assessed for a wide range of spatial frequencies.

Off-axis electron holograms and defocus series of bright-field TEM images were recorded at an accelerating voltage of $300 \mathrm{kV}$ using an FEI Titan 80-300 TEM equipped with an electron biprism and a Gatan imaging filter with a $2048 \times 2048$ pixel charge-coupled device camera. A $10 \mathrm{eV}$ energy-selecting slit was inserted and centered on the zero-loss peak for both off-axis and in-line electron holography in order to reduce the contribution of inelastically scattered electrons.

For off-axis electron holography, the biprism voltage was set to $80.5 \mathrm{~V}$ ( $0.45 \mathrm{~nm}$ fringe spacing) for the $\mathrm{MgO}$ cubes and to $139 \mathrm{~V}\left(0.53 \mathrm{~nm}\right.$ fringe spacing) for the Fe-filled $\mathrm{C}$ nanospheres. Holografree software ${ }^{23}$ was used for off-axis electron hologram reconstruction. 
For in-line electron holography, focal series were recorded using the FWRWtools ${ }^{24}$ plugin for Digital Micrograph, which automates image acquisition and compensates for specimen drift. The nominal defocus values were set according to the formula

$$
\Delta f_{n}=\Delta_{0} \frac{|n|^{p} n}{|n|},
$$

with $\Delta_{0}=400 \mathrm{~nm}$ and $n=\ldots-2,-1,0,1,2 \ldots$ If $p=2$ or $p=3$, phase information can be sampled very efficiently for both low and high spatial frequencies ${ }^{22}$. For the $\mathrm{MgO}$ cubes, the defocus values spanned the range -260 to $330 \mathrm{~nm}$, with $40 \mathrm{~nm}$ defocus steps and linear increments $(p=1)$. For the Fe-filled $C$ nanospheres, the defocus values spanned the range -3.6 to $3.6 \mu \mathrm{m}$, with $600 \mathrm{~nm}$ defocus steps and linear increments $(p=1)$.

Exit surface wave functions were reconstructed using the flux-preserving imaging model and corresponding reconstruction algorithm ${ }^{21}$, combined with gradient flipping (GF-FRWR). Details of the reconstruction procedure are given in the supplemantary information (SI).

In order to monitor convergence of the reconstruction, a mismatch or residual $M$ value was computed between images simulated from the reconstructed wave function and the experimental data ${ }^{25}$, according to the expression

$$
M=\frac{\sum\left|I_{\sin }-I_{\mathrm{sxp}}\right|}{\sum I_{\mathrm{exp}}} .
$$

\section{lii. Results And Discussion}

Figure 2 shows phase images of $\mathrm{MgO}$ cubes obtained using the conventional iterative flux-preserving inline holography reconstruction algorithm ${ }^{21}$ (Fig. 2a), the same flux-preserving approximation with gradient flipping applied (Fig. 2b) and off-axis holography (Fig. 2c). The phase obtained using the conventional in-line reconstruction algorithm varies over the range -2 to $6 \mathrm{rad}$, which is $\sim 50 \%$ lower than that recovered using off-axis holography. The rapid decrease in phase for iterative reconstruction just outside the $\mathrm{MgO}$ cube (Fig. 2a and the red profile in Fig. 2d) results from missing low spatial frequencies. Figure $2 b$ and the corresponding line scan in Fig. $2 d$ show that gradient flipping prevents artifacts at the edges of the $\mathrm{MgO}$ cube and results in a homogeneous background. The agreement between Figs. $2 \mathrm{~b}$ and $2 \mathrm{c}$ is satisfying. The remaining difference of $\sim 20 \%$ between the GF-FRWR and off-axis results may be attributed partially to imperfect energy filtering during the acquisition of the focal series. It should be noted that off-axis electron holography inherently filters out inelastic scattering ${ }^{26,27}$, whereas in-line electron holography has to rely on the use of an energy filter. In order to reduce the influence of inelastic scattering, the imaging model used here calculates and subtracts the inelastic contribution to first order. 
The power spectra are shown in Fig. 2e highlight the differences in information transfer between the three methods. The phase resolutions obtained using in-line and off-axis electron holography are $0.34 \mathrm{~nm}$ and $1.2 \mathrm{~nm}$, respectively. (The fields of view are identical, but a reciprocal space mask was applied during the reconstruction of the wave function from the off-axis data in order to achieve a reasonable signal to noise ratio). The power spectra show that the GF-FRWR algorithm recovers information reliably over distances of up to $\sim 80 \mathrm{~nm}$, whereas the FRWR reconstruction is only reliable over distances of up to $\sim 30$ $\mathrm{nm}$.

Although the GF-FRWR algorithm did not recover phase differences reliably over distances larger than $\sim 80 \mathrm{~nm}$, the present example showed an improvement over the conventional reconstruction algorithm, with dramatically reduced artifacts at the edges of the particles originating from missing low spatial frequencies. The reconstructed phase images look much closer to results obtained using off-axis holography while retaining the superior spatial resolution of focal series reconstruction. This approach may help in the measurement of quantities such as variations in mean inner potential across short distances.

In the second example, results obtained from Fe-filled $\mathrm{C}$ nanospheres provide similar conclusions to those obtained from MgO cubes. Phase images obtained using conventional in-line (FRWR) reconstruction, GFFRWR, and off-axis electron holography are shown in Figs. 3a, 3b, and 3c, respectively. Conventional FRWR recovers phase differences up to $\pi$ radians, whereas off-axis reconstruction shows that the phase spans a range of $4.12 \pm 0.144$ radians. The discrepancy is reduced to $~ 80 \%$ of the off-axis phase when GF-FRWR is applied. The large phase variations at the edges, which are associated with missing low spatial frequencies, are prominent in the conventional FRWR reconstruction in Fig. 3d (red line), but are significantly reduced when using gradient flipping. In Fig. 3e, power spectra are shown for the three reconstruction schemes, confirming that low spatial frequency information obtained using the GF-FRWR algorithm is much closer to that obtained using off-axis holography than FRWR.

We now discuss the effect of the parameter $r_{\mathrm{C}}$ in Eq. (SI1) in the SI. This parameter determines the real space resolution above which gradient flipping is applied. Phase information for distances below $r_{\mathrm{c}}$ is determined by the iterative FRWR reconstruction algorithm, with GF only affecting relative phases across greater distances. Figure 4 shows phase images and profiles reconstructed using different values of $r_{\mathrm{c}}$ for the Fe-filled $\mathrm{C}$ nanospheres. Increasing $r_{\mathrm{c}}$ initially improves the contrast in the phase. Figures $4 \mathrm{a}$ to $4 \mathrm{~d}$ show that the lowest contrast is obtained for $r_{\mathrm{C}}=5 \mathrm{~nm}$. The highest contrast is obtained with $r_{\mathrm{c}}$ set to 25 $\mathrm{nm}$ (Fig. 4g). Increasing $r_{\mathrm{c}}$ further does not improve the contrast.

If $r_{c}$ is set to be much higher than the lateral coherence length of the incident electron wave function, then reconstruction proceeds as if no gradient flipping had been applied since the exponential in Eq. (SI1) only includes very low spatial frequencies. For this reason, dark features appear around the particle in Fig. $4 f$ when the threshold value is too high. 
The $M$ value defined in Eq. (2), which measures the mismatch between experimental and simulated images during reconstruction, is also smallest at $r_{\mathrm{c}}=25 \mathrm{~nm}$ (Fig. 4g). Figure $4 \mathrm{~h}$ shows the dependence of $M$ on $r_{\mathrm{c}}$. The minimum value is obtained when the contrast is maximized at $r_{c}=25 \mathrm{~nm}$, indicating that $M$ is likely to be a suitable figure of merit for optimizing $r_{c}$

The profile taken from the GF-FRWR reconstruction shows an offset in phase between vacuum regions on opposite sides of the core-shell particle (Fig. 4i). This problem arises because the vacuum regions are not connected. Ideally, both sides of the particle should have the same phase shift. We again observe minimum phase offset differences for minimum $M$ and highest contrast, i.e., where $r_{\mathrm{c}}$ is $25 \mathrm{~nm}$.

FIG. 5 Phase images of Fe-filled $\mathrm{C}$ nanospheres reconstructed using phase prediction thresholds of a) 0.01 , b) 0.05 , c) 0.1 , d) 0.25 , e) 0.31 , f) 0.4 , g ) 0.5 , h) 1.0 and i) 0.20 rad. j) Phase prediction threshold $v s$ $M$ value (mismatch between simulated and experimental images) and phase contrast. $k$ ) Magnified version of j). l) Line profiles extracted from the region marked in a) for phase images a) to i).

When the PPT value was set to 0.4 or 0.5 rad (Figs. $5 f-5 g$ ), reconstruction artifacts became obvious. For the more extreme case of PPT $=1 \mathrm{rad}$, the phase could no longer be reconstructed (Fig. $5 \mathrm{~h}$ ). For both this data set and PPT $=0.31$ rad, the reconstruction diverged when the number of iterations exceeded 2500 . For PPT $=0.20$ rad, the algorithm diverged when it was run for more than 4000 iterations. This behavior seems to be related to the way in which the phase is treated in the padded area outside the field of view, where experimental intensity data are available. Since it is not practical to use such large numbers of iterations, this is not a limitation for the application of the algorithm. After determination of the ideal values for $r_{C}$ and PPT using a small number of iterations, the iteration number was increased to obtain a minimum value of the mismatch $M$, which was closest to the actual object wave.

The individual and combined effects of phase prediction and gradient flipping are shown in Fig. 6 . The comparison shows that phase prediction increases the contrast significantly and can recover much lower spatial frequencies in the phase than the iterative nonlinear flux-preserving focal series reconstruction algorithm alone, even when gradient flipping is turned on. Figure $6 \mathrm{a}$ and the red line profile in Fig. $6 \mathrm{~d}$ show that phase prediction results in extended contrast. The same figures show significant edge artifacts associated with missing low spatial frequencies when gradient flipping is not used. Gradient flipping corrects for the missing low spatial frequency information by finding a phase image that is consistent with the experimental data and sparse in its gradient. The blue profile in Fig. $6 \mathrm{~d}$ shows a constant phase profile in the vacuum region, where no phase variations are expected. Although both gradient flipping and phase prediction individually have strong effects on the reconstructed phase, the most significant improvement results from the use of gradient flipping and phase prediction together. Figure $6 \mathrm{c}$ and the green profile in Fig. $6 \mathrm{~d}$ show how edge artifacts are then significantly reduced.

\section{Iv. Conclusions}


We have shown that a charge flipping algorithm, which is successful in solving the crystallographic phase problem in diffraction for finding solutions that are sparse in the space of charge density (X-ray diffraction) or electrostatic potential (electron diffraction), can be adapted to recover phase images that are sparse in their gradient domain using a nonlinear flux-preserving in-line holography reconstruction algorithm. A nonlinear iterative gradient flipping reconstruction algorithm has been developed that overcomes the limitations of conventional reconstruction algorithms due to both an incoherent background in the image intensities and poor encoding of low spatial frequency phase information. Its ability to recover low spatial frequency phase information is demonstrated for two experimental test cases, and the results are compared with off-axis electron holography. For these test cases, the combination of gradient flipping with phase prediction, incoherent background subtraction, and a nonlinear flux-preserving iterative reconstruction scheme provide semi-quantitative phase maps that recover approximately $80 \%$ of the phase contrast reconstructed using off-axis electron holography.

\section{Declarations}

\section{ACKNOWLEDGMENTS}

The authors are grateful to John Bonevich for offering free public use of HolograFREE off-axis electron holography reconstruction software and to N.Y. Jin-Phillipp and B. Ozdol for providing samples. C.T.K. thanks the Deutsche Forschungsgemeinschaft (DFG, German Research Foundation - project numbers 182087777 - SFB 951 and KO 2911/12-1) for financial support. The research leading to these results received funding from the European Union's Horizon 2020 Research and Innovation Programme (Grant No. 823717, project “ESTEEM3").

\section{References}

1. Gabor, D. A New Microscopic Principle | Nature. Nature 161, 777-778 (1948).

2. Tonomura, A. Applications of electron holography. Rev. Mod. Phys. 59, 639-669 (1987).

3. Lichte, H. et al. Electron Holography: Applications to Materials Questions. Annu. Rev. Mater. Res. 37, 539-588 (2007).

4. Lehmann, M. \& Lichte, H. Tutorial on off-axis electron holography. Microsc Microanal 8, 447-466 (2002).

5. Gan, Z. et al. Direct Mapping of Charge Distribution during Lithiation of Ge Nanowires Using Off-Axis Electron Holography. Nano Lett. 16, 3748-3753 (2016).

6. Hÿtch, M. et al. Dark-Field Electron Holography for Strain Mapping. in Transmission Electron Microscopy in Micro-Nanoelectronics 81-106 (John Wiley \& Sons, Ltd, 2013). doi:10.1002/9781118579022.ch4.

7. Koch, C. T., Özdöl, V. B. \& van Aken, P. A. An efficient, simple, and precise way to map strain with nanometer resolution in semiconductor devices. Appl. Phys. Lett. 96, 091901 (2010). 
8. Dunin-Borkowski, R. E. et al. Off-axis electron holography of magnetic nanowires and chains, rings, and planar arrays of magnetic nanoparticles. Microscopy Research and Technique 64, 390-402 (2004).

9. Rau, W. D., Schwander, P., Baumann, F. H., Höppner, W. \& Ourmazd, A. Two-Dimensional Mapping of the Electrostatic Potential in Transistors by Electron Holography. Phys. Rev. Lett. 82, 2614-2617 (1999).

10. Tonomura, A., Matsuda, T., Endo, J., Todokoro, H. \& Komoda, T. Development of a Field Emission Electron Microscope. J Electron Microsc (Tokyo) 28, 1-11 (1979).

11. Leith, E. N. \& Upatnieks, J. Reconstructed Wavefronts and Communication Theory*. J. Opt. Soc. Am., JOSA 52, 1123-1130 (1962).

12. Latychevskaia, T. \& Fink, H.-W. Solution to the Twin Image Problem in Holography. Phys. Rev. Lett. 98, 233901 (2007).

13. Möllenstedt, G. \& Düker, H. Fresnelscher Interferenzversuch mit einem Biprisma für Elektronenwellen. Naturwissenschaften 42, 41-41 (1955).

14. Teague, M. R. Deterministic phase retrieval: a Green's function solution. J. Opt. Soc. Am., JOSA 73, 1434-1441 (1983).

15. Gureyev, T. E. Composite techniques for phase retrieval in the Fresnel region. Optics Communications 220, 49-58 (2003).

16. Gerchberg, R. W. \& Saxton, W. O. A Practical Algorithm for the Determination of Phase from Image and Diffraction Plane Pictures. Optik 35, (1972).

17. Ophus, C. \& Ewalds, T. Guidelines for quantitative reconstruction of complex exit waves in HRTEM. Ultramicroscopy 113, 88-95 (2012).

18. Ozsoy-Keskinbora, C., Boothroyd, C. B., Dunin-Borkowski, R. E., van Aken, P. A. \& Koch, C. T. Hybridization approach to in-line and off-axis (electron) holography for superior resolution and phase sensitivity I Scientific Reports. Scientific Reports volume 4, (2014).

19. Parvizi, A., Broek, W. V. den \& Koch, C. T. Recovering low spatial frequencies in wavefront sensing based on intensity measurements. Advanced Structural and Chemical Imaging 2, 3 (2016).

20. Parvizi, A., Broek, W. V. den \& Koch, C. T. Gradient flipping algorithm: introducing non-convex constraints in wavefront reconstructions with the transport of intensity equation. Opt. Express, OE 24, 8344-8359 (2016).

21. Koch, C. T. A flux-preserving nonlinear in-line holography reconstruction algorithm for partially coherent electrons. Ultramicroscopy 108, 141-150 (2007).

22. Koch, C. T. Towards full-resolution in-line electron holography. Micron 63, 69-75 (2014).

23. Bonevich, J. HolograFREE software. HolograFREE software http://www.nist.gov/mml/msed/functional_nanostructure/electron_holography.cfm.

24. Koch, C. T. FRWRtools plugin. FRWRtools plugin https://www.physics.huberlin.de/en/sem/software/software_frwrtools (2016). 
25. Brünger, A. T. Free R value: a novel statistical quantity for assessing the accuracy of crystal structures., Published online: 30 January 1992; / doi:10.1038/355472a0 355, 472-475 (1992).

26. Lichte, H., Geiger, D. \& Linck, M. Off-axis electron holography in an aberration-corrected transmission electron microscope. Philosophical Transactions of the Royal Society A: Mathematical, Physical and Engineering Sciences 367, 3773-3793 (2009).

27. Ozsoy-Keskinbora, C., Boothroyd, C. B., Dunin-Borkowski, R. E., van Aken, P. A. \& Koch, C. T. Mapping the electrostatic potential of Au nanoparticles using hybrid electron holography. Ultramicroscopy 165, 8-14 (2016).

28. Oszlányi, G. \& Sütő, A. Ab initio structure solution by charge flipping. Acta Cryst A 60, 134-141 (2004).

\section{Figures}

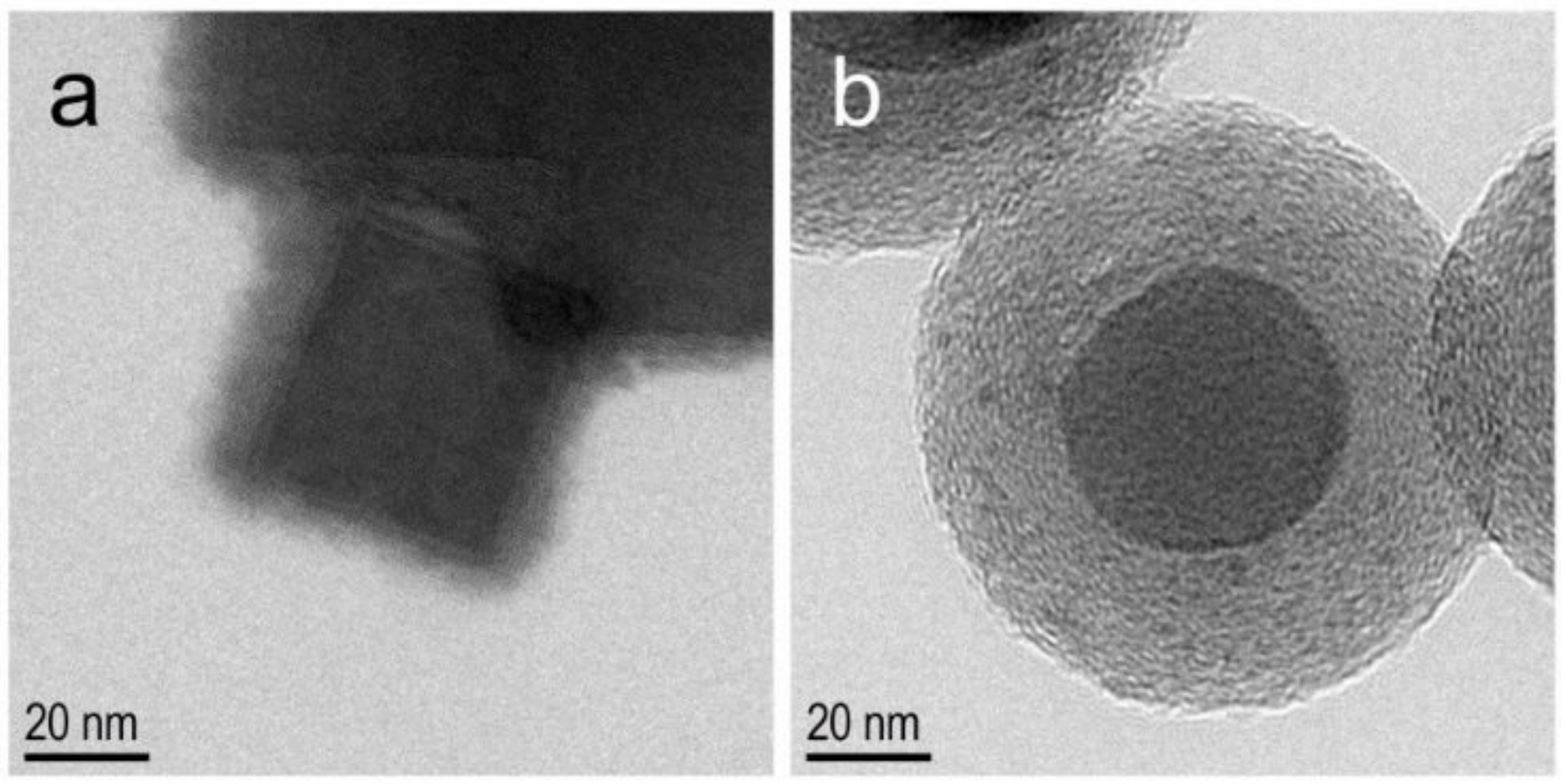

\section{Figure 1}

Bright-field TEM images of a) MgO cubes; b) Fe-filled C nanospheres.

\section{Figure 2}


Phase images of $\mathrm{MgO}$ cubes reconstructed using a) FRWR, b) GF-FRWR, and c) off-axis electron holography. d) Line profiles extracted from the three phase reconstructions. e) Radially-averaged power spectra of the three phase images.

\section{Figure 3}

Phase images of Fe-filled C nanospheres were reconstructed using a) FRWR, b) GF-FRWR, and c) off-axis electron holography. d) Line profiles extracted from the three phase reconstructions. e) Radially-averaged power spectra of the three phase images.

\section{Figure 4}

Phase images of Fe-filled C nanospheres reconstructed using a) 5, b) 10, c) 15, d) 20, e) 30, f) 40, and g ) $25 \mathrm{~nm}$ threshold values. h) Threshold $v s \mathrm{M}$ value (mismatch between simulated and experimental images). i) Line profiles of a) to g) from the region marked in a).

\section{Figure 5}

Phase images of Fe-filled $\mathrm{C}$ nanospheres reconstructed using phase prediction thresholds of a) 0.01 , b) 0.05 , c) 0.1 , d) 0.25 , e) 0.31 , f) 0.4 , g ) 0.5 , h) 1.0 and i) 0.20 rad. j) Phase prediction threshold $v s \mathrm{M}$ value (mismatch between simulated and experimental images) and phase contrast. $\mathrm{k}$ ) Magnified version of j). I) Line profiles extracted from the region marked in a) for phase images a) to i).

\section{Figure 6}

Comparison between individual and combined effects of gradient flipping and phase prediction. a) Phase reconstruction with gradient flipping (GF) off and phase prediction (PP) on. b) Phase reconstruction with GF on and PP off. c) Phase reconstruction with GF on and PP on. d) Line profiles extracted along the lines marked in the phase maps. 